\title{
L'enseignement universitaire à distance en Tunisie : promesses et obstacles à son adoption
}

\section{Web-based university education in Tunisia: promises and obstacles to its adoption}

\author{
Khaoula Ouni Ghobtane ${ }^{1}$, Henia Ben Amor ${ }^{2}$ \\ ${ }^{1}$ Laboratoire Entreprise\& Recherche Marketing, Université Tunis El Manar, Tunisie, kouni84@gmail.com. \\ ${ }^{2}$ Laboratoire Théorie économique, modélisation et applications, Université Tunis El Manar, Tunisie, \\ henia.benamor@recherche.uma.tn
}

RÉSUMÉ. Cet article examine l'impact de la digitalisation de l'enseignement supérieur sur les inégalités sociales pendant la crise sanitaire (COVID-19). Après avoir rappelé l'expansion de l'enseignement universitaire à distance ainsi que l'évaluation de l'efficacité de ce nouveau paradigme d'apprentissage, nous approfondissons le lien entre l'enseignement à distance et l'inégalité sociale. S'appuyant sur une étude qualitative exploratoire auprès de trente enseignants universitaires tunisiens, les résultats montrent que la résistance aux changements et la réticence, l'absence de lieu propice de travail et la lenteur de connexion Internet constituent les principaux obstacles à l'adoption de cette nouvelle façon d'apprentissage. De plus, notre étude montre que $60 \%$ des enseignants considèrent que l'enseignement à distance présente un puissant accélérateur des inégalités sociales tant pour les enseignants que pour les étudiants.

ABSTRACT. This article examines the impact of the digitalization of higher education on social inequalities during the health crisis (COVID-19). After a reminder on the expansion of distance education as well as the evaluation of the effectiveness of this new learning paradigm, we go into more depth on the link between distance education and social inequality. Based on an exploratory qualitative study of thirty Tunisian university teachers, the results show that resistance to change and reluctance, the lack of a suitable place to work and the slowness of Internet connection constitute the main obstacles to the adoption of this new way of learning. In addition, our study shows that $60 \%$ of teachers consider that distance education is a cruel accelerator of social inequalities for both teachers and students.

MOTS-CLÉS. Technologies d'information et de la télécommunication, Enseignement à distance, crise sanitaire, inégalités sociales, difficultés.

KEYWORDS. Information and telecommunication technologies, distance learning, health crisis, social inequalities, difficulties.

\section{Introduction :}

L'arrivée des nouvelles technologies de l'information et de la communication (NTIC) transforme considérablement le paysage informationnel, notamment au niveau des pratiques d'apprentissage. Le confinement sanitaire général imposé en Tunisie le mois de mars 2020 a favorisé l'expérimentation accélérée de ces nouvelles pratiques d'apprentissage au sein des universités tunisiennes. En effet, les impacts de cette crise pandémique ont été très forts, l'enseignement a radicalement changé, avec l'essor sans précédent de l'éducation en ligne, où l'apprentissage est dispensé à distance à travers des plateformes numériques.

Ce passage brusque à l'enseignement en ligne, sans préparation préalable technique et pédagogique, a bouleversé le système éducatif dans les universités tunisiennes qui ont été mises au défi de s'ajuster très rapidement à ce nouveau contexte pour faire face à l'urgence. Cette situation fonde notre question centrale qui est la suivante : Quels sont les changements induits par la crise de la Covid-19 sur l'enseignement supérieur dans les universités tunisiennes ?

La présente recherche s'intéresse à l'adoption de l'innovation technologique de l'information et de la communication dans le secteur éducatif suite à la crise sanitaire. Notre étude s'inscrit ainsi dans le cadre de la théorie diffusionniste de l'innovation de [ROG 95, ROG 03]. Cette théorie fait référence à la propagation de nouvelles idées et de nouveaux comportements dans un groupe social et permet de comprendre les facteurs entrant en jeu lors de l'adoption des innovations dans une communauté. 
En effet, certains individus sont plus rapides que d'autres à adopter des innovations. En se référant aux travaux de [ROG 03], la probabilité d'adoption d'une innovation dépend essentiellement de la cible, des obstacles environnementaux, des réseaux de communication et des attributs de l'innovation, tels que : l'avantage relatif d'une innovation, la compatibilité de l'innovation avec les valeurs des utilisateurs potentiels, leurs besoins et leurs expériences antérieures, la complexité de l'innovation, la possibilité de l'essayer et finalement son caractère observable.

Le modèle de la diffusion des innovations ne s'intéresse pas aux conditions de la création d'une innovation, mais plutôt il explique comment une innovation peut se diffuser auprès d'un groupe d'utilisateurs. Ce modèle a rencontré un grand succès, plus de 70 articles traitant des nouvelles technologies de l'information et de la communication concernent la théorie diffusionniste de l'innovation [PRE 95]. Toutefois, le modèle diffusionniste manque de spécificités vu qu'il s'applique à tous types d'innovations, alors que l'adoption de certaines innovations peut présenter des caractéristiques très particulières [CHA 97, LYY01].

L'avantage principal de ce modèle réside dans une description dynamique du mécanisme d'adoption d'une innovation [VEN02]. Selon [ROG 95], l'adoption d'une innovation se présente essentiellement comme le processus d'acceptation d'une nouveauté par une unité décisionnelle qui évolue dans un contexte social particulier. Pour lui, l'adoption d'une innovation est un processus strictement individuel qui se déroule en cinq phases allant de la connaissance de l'innovation à la confirmation ou au rejet de l'adoption, alors que dans son ensemble elle concerne le groupe, composé principalement d'un agglomérat d'individualités [HAM 11].

Quoique ce modèle prenne en considération des éléments relatifs à la perception individuelle des attributs portés par une innovation, il est principalement développé afin d'expliquer le comportement d'adoption d'une innovation par un système social [MOO 91]. [ROG 95] définit un système social comme un groupe d'unités reliées entre elles et engagées dans des activités communes afin d'atteindre des objectifs communs. Dans le cadre de notre étude ce groupe est formé par des enseignants universitaires dont le but est l'enseignement à distance durant la crise sanitaire.

Dans la littérature sur l'adoption de l'innovation dans le domaine de l'enseignement, [CAN19] applique le modèle de [ROG 03] pour étudier les facteurs d'adoption ou de rejet des technologies chez les professeurs. Il prouve que les professeurs préfèrent les outils plus conventionnels et éprouvent une certaine résistance aux technologies les plus récentes. Dans la même veine, [BEA, 16] se réfère au même modèle afin d'étudier les conditions de réussite de l'implantation d'une innovation en formation à distance pour le développement de l'enseignement supérieur.

La présente recherche a pour objectif d'explorer l'éducation en temps de Covid-19 dans les universités tunisiennes, de dévoiler les obstacles rencontrés et d'étudier l'impact de la digitalisation de l'éducation sur l'inégalité sociale et l'équité d'apprentissage. La première partie de cet article est consacrée à l'état des lieux de l'EAD en Tunisie et à la revue de la littérature. Plus particulièrement, nous mettons en évidence, l'expansion de l'EAD ainsi que son efficacité et nous creusons le sujet de l'EAD et l'inégalité sociale. La deuxième partie revient sur les considérations méthodologiques et la troisième partie traite les résultats de la recherche.

\section{L'état des lieux de I'EAD en Tunisie :}

Dans les pays industriels, l'enseignement à distance a longtemps été considéré comme une éducation de remplacement pour les personnes à faible revenu qui n'ont pas reçu une éducation adéquate mais qui ont besoin d'une qualification spécifique pour trouver leur place sur le marché du travail [ADE11]. Cependant, bien qu'il y ait eu des développements particulièrement importants au cours des 20 dernières années, personne ne peut nier que dans de nombreux pays dits du Sud, dont la Tunisie, l'enseignement supérieur est confronté à des difficultés énormes, de même que la mise en place de projets d'enseignements à distance. 


\subsection{Les obstacles de la mise en place de L'EAD:}

Il existe plusieurs obstacles pour la mise en place d'un projet d'EAD dans les pays du Sud. En effet, la majorité des pays en développement ont rencontré une panoplie de freins pour le développement de l'EAD, dont notamment la Tunisie.

Le manque de fonds constitue l'un des principaux obstacles du développement de l'enseignement dans les pays de Sud. Par comparaison, la part du PIB allouée à l'enseignement et à la recherche scientifique est presque quatre fois plus faible que celle dans les pays industriels [KAR 12].

Les crises économiques vécues par ces pays constituent également un grand handicap qui retarde le progrès du secteur éducatif. En réalité, le budget alloué à l'enseignement et à la recherche scientifique dans les pays du Sud limite, dans une grande mesure, les possibilités d'investissements dans le domaine de l'enseignement [POU10].

Au-delà des problèmes économiques auxquels les responsables politiques doivent faire face, les crises politiques se sont aussi directement répercutées sur le secteur éducatif. Le développement de l'EAD n'est pas une priorité majeure pour ces pays en crise et les programmes de formation sont rares et souvent mal coordonnés. Un faible intérêt de la part des gouverneurs est alors porté pour le développement du système éducatif.

Quand on entreprend d'évoquer les obstacles du développement de l'EAD dans les pays du Sud, il convient de ne pas oublier l'infrastructure des technologies de l'information et de la communication(TIC) dans les universités qui présente un certain nombre de lacunes techniques très évidentes [CAC 06, BON 10]. En effet, le débit de connexion Internet est souvent insuffisant et de mauvaise qualité. Les universités souffrent également d'une pénurie récurrente de matériel informatique et de logiciels, ainsi que de confidentialité, de sécurité des données informatiques et des attaques de virus réduisant d'autant plus les capacités d'accès à l'Internet.

Selon [CHR 12], bien que l'accès à Internet s'améliore dans les pays en développement, les infrastructures sont encore insuffisantes pour permettre d'envisager une utilisation massive du e-Learning. Par rapport aux pays développés, l'accès à l'Internet dans les pays en développement est encore très limité. Cela met en évidence la fracture numérique qui sépare les pays les plus développés des pays en développement. De plus, les possibilités d'accès à l'Internet pour les pays en développement se caractérisent par des variations importantes entre les pays et même, au sein d'un même pays, entre les régions.

Les problèmes de maintenance, de budget de fonctionnement ainsi que la formation insuffisante des utilisateurs qui rendent difficile la mise en œuvre de divers projets de TIC dans l'éducation constituent aussi des problèmes majeurs pour l'amélioration de l'apprentissage en ligne.

Actuellement, professeurs et instituteurs, dont les salaires avaient déjà fondu dans les pays du Sud, ne peuvent vivre avec des salaires aussi bas. En Tunisie, la situation matérielle du professeur d'université s'est dégradée, de sorte qu'il y a un décalage de $49 \%$ entre le taux de variation des salaires et du PIB [MAR18].

Plusieurs observateurs remarquent le manque criant de ressources financières et humaines dans le secteur éducatif, le manque de matériel pédagogique et informatique élémentaire, le faible niveau de formation des enseignants, le manque d'efficacité d'universités paupérisées et les classes surchargées [POU10].

Selon une étude de l'Association tunisienne des grandes écoles (Atuge), 4200 professeurs d'université ont quitté la Tunisie après la révolution. Ces derniers quittent le pays pour s'installer dans des pays connus par des environnements plus sûrs où les salaires correspondent à leurs qualifications. 
L'étude revient, entre autres, sur la dévaluation de la recherche scientifique à l'université tunisienne. Cela se traduit par la révision à la baisse du budget de la recherche scientifique de $75 \%$ entre 2016 et 2018 [MAR18].

Il importe que durant ces dernières années, les universités tunisiennes risquent de devenir des usines pour les chômeurs et ceci est dû principalement à l'absence de formation adéquate avec le marché du travail ainsi que la présence de formations professionnelles embryonnaires. En effet, selon l'institut national des statistiques, le taux de chômage pour les diplômés de l'enseignement supérieur a augmenté durant le deuxième trimestre de 2020 pour atteindre $31,2 \%$, de plus $37.6 \%$ des jeunes diplômés sont surqualifiés par rapport au travail effectué.

\subsection{L'émergence de l'EAD en Tunisie}

Le secteur de l'enseignement supérieur tunisien a déployé des efforts sérieux pour la mise en œuvre d'un projet d'enseignement à distance au sein des établissements universitaires. En effet, de nouvelles méthodes d'enseignement émergent et incluent de nouveaux contextes de travail et de nouvelles modes d'apprentissage.

Il est important de souligner que l'enseignement à distance existe en Tunisie depuis 1983. L'origine de cette expérience est de compenser le manque d'enseignants qualifiés, notamment en introduisant des procédures de formation accélérées pour ceux qui travaillent dans des établissements d'enseignement éloignés de la capitale. C'est pourquoi l'Institut supérieur pour l'éducation et la formation continue (ISEFC) a été fondé. En outre, l'Université Virtuelle de Tunisie (UVT) est chargée de faire la transition vers l'enseignement à distance pour les étudiants des établissements d'enseignement supérieur, avec une formation basée sur la maîtrise nécessaire des technologies de l'information et de la communication. En raison de la volonté institutionnelle, ce nouvel organe a été créé par le décret $\mathrm{n}{ }^{\circ}$ 112-02 du 28 janvier 2002, dans le cadre de la politique de modernisation du secteur de l'enseignement supérieur tunisien qui utilise les technologies de l'information et de la communication comme outils de travail et de recherche.

Depuis 2003, l'Université Virtuelle de Tunisie a lancé son premier programme dans les instituts supérieurs des études technologiques (ISET). Il consiste à fournir aux étudiants des ressources d'apprentissage à distance, en fonction de leurs domaines et niveaux d'études. La création de cet organisme universitaire (ISET) remonte à 1995. Il s'agit d'instituts scientifiques et techniques chargés de la formation des techniciens supérieurs. L'émergence de nouvelles demandes et besoins dans tous les secteurs commerciaux en général et dans les secteurs secondaires et tertiaires en particulier, a incité l'Etat tunisien à créer ce nouvel établissement d'enseignement supérieur, dans le but de satisfaire les besoins de l'économie nationale et internationale, à partir de la qualification des ressources humaines des entreprises tunisiennes.

\section{Cadre théorique de la recherche}

\subsection{L'EAD : Un mode d'enseignement en expansion}

L'enseignement à distance est l'un des secteurs universitaires qui croit à un rythme très rapide. Depuis le début du XXe siècle, il est progressivement devenu une partie importante des institutions académiques et il a joué un rôle important dans le développement des pratiques d'enseignement universitaire.

Tout au long de l'histoire, l'impact de la technologie sur l'éducation et la formation a joué un rôle crucial. L'imprimerie, l'écriture, l'audiovisuel ainsi que la micro-informatique ont profondément changé les méthodes classiques d'éducation et de formation.

Les premiers cours d'enseignement à distance ont été développés aux États-Unis au milieu du XIXe siècle. Des décennies plus tard, les universités se sont tournées vers l'enseignement à distance. 
En raison de l'essor des technologies de l'information et de la communication ainsi que le développement des plates formes numériques de l'éducation, l'enseignement à distance a franchi le seuil de la cinquième génération. Premièrement, l'imprimé marque le début de l'enseignement à distance et constitue la base de l'étude par correspondance. C'est le principal outil d'enseignement pour laquelle le support papier est le mode de communication dominant.

Le premier cours par correspondance a été lancé en Angleterre en 1840, année de la parution du timbre-poste, et marque le début de l'enseignement à distance, dispositif assez ancien et largement développé en Europe, puis dans le reste du monde. Il est important de noter que l'enseignement à distance dans sa forme la plus ancienne était lié à une technologie, le timbre-poste, qui rendait les échanges plus fiables d'une certaine manière [BLA 04]. L'enseignement à distance s'est développé plus tard et il est devenu une « seconde chance »permettant aux adultes qui ne pouvaient pas terminer leurs études secondaires ou supérieures d'achever leurs apprentissages.

L'ère du multimédia a débuté dans les années 1960, caractérisée par l'utilisation de différents médias (presse écrite, radio, télévision, vidéo) complémentaires et coordonnés en vue d'un objectif éducatif commun. La deuxième génération d'enseignement à distance se caractérise par l'utilisation de la télévision et surtout de la vidéocassette comme support de contenu. Pour renforcer l'efficacité des systèmes éducatifs, perçus comme peu performants, l'éducation explore les possibilités de différents médias, télévision, radio, cassettes audio et est rapidement qualifiée de technologies éducatives ou de techniques éducatives modernes.

Cette deuxième génération d'enseignement à distance s'est développée dans un contexte dominé par des concepts éducatifs «comportementaux». L'imprimé reste le support de base, mais l'industrie audiovisuelle joue un rôle de plus en plus important dans l'éducation. L'interaction se limite encore à la correction des travaux par correspondance et parfois par téléphone entre l'étudiant et le tuteur [KEE96].

Dans les années 1980, avec la naissance de la micro-informatique puis de la télématique, commence l'ère moderne, celle de l'Internet, de l'hypermédia et du multimédia polyvalent. Les progrès des microordinateurs et des télécommunications, qui ont marqué cette époque, ont permis à l'enseignement à distance de mettre en œuvre une nouvelle mutation, celle de l'interaction: chaque élève interagit avec son professeur et ses pairs par visioconférence, par e-mail et lors de discussions en ligne .Il exploite également les ressources pédagogiques de l'Internet, il s'auto-évalue sur Internet en devenant très autonome. Ces interactions réduisent considérablement les taux d'abandon. De plus, les technologies éducatives se sont diversifiées: imprimé, audiovisuel, multimédia, interaction par téléphone, e-mail, Internet, visioconférence, etc.

La troisième génération d'enseignement à distance a émergé dans un contexte influencé par les conceptions « constructivistes $»$ de l'enseignement et a permis d'utiliser des technologies interactives pour l'apprentissage collaboratif, la reformulation des concepts étudiés par l'apprenant, des autoévaluations anonymes et instantanées, etc.

Après l'enseignement par correspondance, l'enseignement à distance assisté par l'audiovisuel et l'enseignement à distance assisté par l'ordinateur, les technologies de l'information et de la communication (TIC) franchissent un nouveau pas vers cette méthode d'enseignement [POW02].

En effet, Les TIC ont permis à l'EAD de franchir le seuil de sa 4ème génération grâce aux multiples possibilités que permet le Web. Selon [RUM97] et [DAN96] cités par [POW02], plus de trois millions d'étudiants s'inscrivent chaque année dans des universités qui offrent des cours à distance, des cours offerts de plus en plus, en totalité ou en partie sur le web.

A l'ère du digital, une cinquième génération d'enseignement à distance a émergé est celle de l'apprentissage numérique « Le digital Learning ». Cette transformation digitale dans le domaine de la 
formation est directement liée à l'émergence des nouvelles technologies numériques dans la vie quotidienne. De l'Internet aux Smartphones, ils sont désormais partout dans la vie personnelle et professionnelle.

L'apprentissage numérique est donc un moyen d'animer le présentiel et d'harmoniser les apprenants et les technologies en proposant une gamme d'outils, de formats et d'approches. L'apprentissage numérique fait référence à toute pratique éducative qui utilise efficacement la technologie pour améliorer l'expérience d'apprentissage d'un apprenant. L'apprentissage numérique couvre tous les types de supports, tels que les ordinateurs, les Smartphones et les tablettes. Il peut également prendre la forme de cours en présentiel. Dans ce cas, l'apprentissage numérique fait partie d'un processus d'apprentissage hybride (formation en ligne et formation en présentiel). C'est ce qu'on appelle «Blended Learning ».

Il existe une panoplie des modalités d'apprentissage numérique, à savoir : le MOOC pour massive online open course, le Microlearning, le social Learning, le vidéo Learning, le Serious Game ou jeu sérieux, la réalité virtuelle ou réalité augmentée ...

\subsection{Revue de la littérature sur l'évaluation de l'efficacité de l'EAD}

Aujourd'hui, l'EAD se développe à un rythme croissant, les technologies utilisées actuellement dans l'apprentissage sont de plus en plus diversifiées, à l'égard des plateformes numériques d'apprentissage, de tableau blanc interactif, de classe virtuelle...

L'importance d'étudier l'efficacité de l'apprentissage à distance à l'ère de la digitalisation de l'éducation est mise en évidence par de nombreux chercheurs, à l'instar de [BAT18] qui considère l'Internet comme le principal moyen de transmission du matériel éducatif. L'auteur déclare que $25 \%$ des établissements universitaires utilisent des technologies qui favorisent la communication synchrone. De même, pour $25 \%$ des universités, le support pédagogique sera transmis par voie électronique. Quant aux technologies moins coûteuses, telles que les réseaux sociaux et les appareils mobiles, elles sont encore sous-utilisées.

Récemment, il y a une controverse, concernant l'impact des progrès technologiques sur l'efficacité de l'apprentissage à distance [ZAN17, FEN 18]. Certains chercheurs rapportent leur contribution positive [LEE17] alors que d'autres soulignent les déceptions face aux dernières technologies [JOH14, MER 14].

La question de l'efficacité des nouveaux modes d'apprentissage est aujourd'hui au cœur des débats entourant l'EAD. Selon [POU15], l'efficacité individuelle est corrélée à la satisfaction de l'étudiant à l'égard des pratiques de l'EAD. Alors que l'efficacité institutionnelle est liée aux taux de réussite ou aux tests de connaissance [ABI13].

En fouillant la littérature portant sur l'efficacité de l'EAD, trois principales théories sont considérées comme les plus crédibles, à savoir : la théorie de la distance transactionnelle de [MOO13], la théorie de [AND13] et celle de [GAR13].

Dans sa théorie de la distance transactionnelle, [MOO13] précise que la transaction est le résultat de l'interaction entre les enseignants et les apprenants et elle se produit dans des lieux spatialement séparés. La distance de la transaction découle des combinaisons changeantes entre le degré de structure, de dialogue (interaction) et d'autonomie de l'apprenant. Cette distance est très importante lorsque le cours a une structure élevée et comporte peu de conversation. Par contre, elle est moins importante lorsqu'il y a une conversation intense et une structure flexible.

Selon [MOO13], c'est la conception pédagogique qui conditionne l'efficacité de l'apprentissage à distance, et non pas les avancées technologies. Ainsi, l'accessibilité aux nouvelles technologies offre aux concepteurs de cours la possibilité de choisir entre différents types de pédagogie. 
De sa part, [AND13] s'est focalisée dans sa théorie sur le concept de l'auto-direction pour évaluer l'efficacité de l'EAD. D'après lui, ce concept englobe deux facettes, à savoir l'apprentissage autonome et l'apprentissage collaboratif. L'auto-direction indique comment les étudiants construisent eux-mêmes leur propre environnement d'apprentissage indépendant ou collaboratif.

Les étudiants contrôlent alors leur mode d'apprentissage dans une transaction, plus ou moins soutenue, impliquant leurs professeurs et leurs collègues. L'EAD offre ainsi de nombreuses opportunités d'interaction avec les acteurs et le contenu des cours, grâce aux dernières avancées technologiques [AND13]. Selon cet auteur, l'efficacité de l'EAD augmenterait en fonction des progrès technologiques.

En se référant à la théorie de l'efficacité de l'apprentissage à distance de [GAR13], nous pourrons penser que la position de ces théoriciens est ambiguë. D'après ces derniers, l'efficacité de l'EAD serait différente selon les progrès technologiques. Toutefois, l'efficacité de l'apprentissage virtuel n'est pas obligatoirement plus élevée lorsque l'utilisation des technologies plus récentes devient inévitable.

Pour ces chercheurs, l'environnement éducatif en ligne facilite la création d'une communauté d'apprentissage. Néanmoins, l'organisation de l'enseignement dans un cadre virtuel exige l'intégration de la technologie dans la pédagogie, ainsi que l'obligation de repenser les stratégies éducatives pour optimiser le potentiel des médias.

Dans l'ensemble, ces positions conceptuelles issues des principales théories de l'apprentissage à distance s'accordent sur l'importance de l'interaction entre l'enseignant et l'apprenant. Cette relation est au cœur de la réussite de l'enseignement virtuel.

\subsection{L'EAD : Un vecteur d'inégalité sociale}

Pour des raisons sanitaires, la majorité des Etats ont choisi comme stratégie pour réduire les contaminations, le confinement total. Cependant, la crise actuelle a révélé et a amplifié les inégalités dans de nombreux domaines de la vie dont le secteur de l'éducation ne fait pas exception. En effet, l'impact de ces circonstances sur la qualité de l'éducation est en cours d'analyse par de nombreux chercheurs de différentes disciplines.

Plusieurs études empiriques sont effectuées dans certains pays pendant et après la période de fermeture des écoles et des universités et, surtout, sur les résultats des interruptions scolaires et universitaires pendant la période de confinement.

La majorité de ces études montrent que la période de confinement est suivie des pertes d'acquis importantes et significatives, qui sont généralement davantage liées aux matières quantitatives et ont tendance à être plus élevées pour les élèves et les étudiants de niveau économique inférieur puisqu'ils ont un accès très inégal à Internet, aux ordinateurs et aux autres outils numériques [ATT 20, CAR 20].

De même, des études récentes révèlent que l'enseignement à distance ne peut que partiellement remplacer l'enseignement en classe et induit une baisse drastique des niveaux intellectuels chez les apprenants [BUR 20, HAE 20, KUH 20, PSA 20, VAN 20]. En outre, l'accès à la technologie et au matériel informatique nécessaire afin d'assurer la continuité de l'apprentissage pendant la fermeture des universités et des écoles n'est pas du tout le même pour tous les apprenants, et avec des conséquences plus durables, l'enseignement à distance peut exacerber les inégalités existantes en matière d'éducation. Les inégalités d'accès à l'éducation à distance en pleine crise de la Coronavirus risquent, de ce fait, d'aggraver la crise d'inégalité d'apprentissage.

\section{Méthodologie de recherche}

Le caractère exploratoire de cette recherche implique l'adoption d'un protocole de recherche qualitative fondé sur des entrevues individuelles semi-directives en face à face d'environ 45 minutes 
chacun. L'entrevue individuelle est en fait un outil privilégié en sciences de gestion car il permet d'explorer les expériences personnelles et les attitudes de la personne envers le phénomène étudié [GAV08].

Notre étude qualitative a été réalisée le mois d'octobre 2020 en s'adressant aux enseignants de certaines universités tunisiennes intervenant en EAD. Préparé à leurs intentions, notre guide d'entretien comprend une série de questions ouvertes ainsi que des questions fermées à choix simple pour identifier le profil de l'interviewé.

Concrètement, notre échantillon est constitué de 30 enseignants impliqués, d'une manière ou d'une autre, en EAD et répartis selon quatre catégories disciplinaires et six statuts professionnels. En effet, les participants à notre investigation ont été recrutés par la méthode d'échantillonnage par convenance. Selon [TED07], l'échantillon de convenance est constitué à partir d'unités de sondage accessibles et coopératives.

A ce niveau, il est à signaler que l'échantillon d'une étude qualitative ne vise pas la représentativité statistique de la population mère [AND05] mais, quand même il doit être " orienté plutôt que pris au hasard » [IFE10] pour assurer une certaine richesse et diversité des points de vue par rapport au thème étudié.

Notre échantillon est ainsi composé de 18 femmes et 12 hommes âgés de 28 à 60 ans et ayant différents grades (Enseignant vacataire, enseignant contractuel, assistant, maitre assistant, maitre de conférence et professeur) et issus de différents catégories disciplinaires (Droit, Economie, Gestion, Sciences politiques, Arts, Langues, Lettres, Sciences humaines, Santé, Sciences et technologies)

Il est à mentionner aussi que le principe de la saturation a été respecté lors de la définition de la taille de l'échantillon. Selon ce principe le processus de constitution de l'échantillon s'arrête lorsque les dernières unités d'observation analysées n'ont apporté aucune nouvelle information pouvant enrichir la recherche [JAO14]. Le tableau ci-dessous présente des informations sur la répartition de nos interviewés.

\begin{tabular}{|c|c|c|}
\hline \multirow{2}{*}{ Catégorie disciplinaire } & \multicolumn{2}{c|}{ Enseignants } \\
\cline { 2 - 3 } & Droit, Economie, Gestion, Sciences politiques & 10 \\
\cline { 2 - 3 } & Arts, Langues, Lettres, Sciences humaines & 9 \\
\cline { 2 - 3 } & Sciences et technologies & 7 \\
\hline Santé & 4 \\
\hline \multirow{2}{*}{ Statut } & Professeur & 3 \\
\hline & Maître de conférences & 5 \\
\hline & Maître Assistant & 7 \\
\hline Assistant & 6 \\
\hline & Enseignant contractuel & 4 \\
\hline
\end{tabular}

Tableau 1. Distribution des enseignants interviewés

\section{Résultats et discussion}

Après avoir enregistré et retranscrit intégralement les entretiens menés dans les 24 heures qui suivent la conversation avec les interviewés, nous avons effectué une analyse des données qualitatives 
à l'aide de la méthode de l'analyse de contenu thématique qui nous permet de découper les informations brutes par thèmes prédéfinis tout en envisageant la possibilité d'apparition de nouveaux thèmes. Le logiciel d'analyse qualitative QSR NVIVO dans sa version 7 nous a servi d'instrument pour emmagasiner les données, les coder et les regrouper en thèmes et sous-thèmes. A l'issue de notre analyse des données, quatre thèmes et vingt-quatre sous-thèmes apparaissent au niveau des verbatim.

\subsection{Expérience avec l'enseignement à distance :}

En se référant aux travaux de [ROG03], il est important de tenir compte des raisons de l'adoption d'une innovation au sein d'une communauté, que ce soit une décision individuelle, collective ou imposée. Les innovations dont l'adoption est imposée, sont probablement les plus rapides à se diffuser, à partir du moment où l'obligation d'adopter l'innovation est respectée par les adoptants concernés. C'est le cas de la décision de l'innovation éducative (à travers l'EAD) qui a été imposée par le ministère de l'enseignement supérieur et de la recherche scientifique en Tunisie pendant la pandémie de la COVID-19 pour assurer la continuité des cours.

Dans la présente recherche, $63,33 \%$ des enseignants déclarent qu'ils ont accepté de s'engager dans une telle démarche puisque la période que nous traversons l'oblige à migrer vers l'enseignement à distance. Par contre, $36,66 \%$ des enseignants refusent la décision du ministère.

\begin{tabular}{|c|c|c|}
\hline \multicolumn{3}{|c|}{$\begin{array}{l}\text { Comment jugez-vous la décision du ministère de l'enseignement supérieur de recourir à l'EAD pendant la } \\
\text { pandémie de Covid-19 ? Est-ce que vous avez pratiqué l'EAD pendant cette période ? }\end{array}$} \\
\hline & Nbre & $\%$ OBS \\
\hline Décision adéquate & 19 & $63,33 \%$ \\
\hline \multicolumn{3}{|c|}{$\begin{array}{l}\text { «Je vois que le ministère de l'enseignement supérieur n'a pas d'autres choix pour garantir la continuité } \\
\text { pédagogique ... Nous devons tout simplement accepter la décision et s'adapter avec le nouveau cadre } \\
\text { d'éducation» (Sujet 29). } \\
\text { «L'EAD est une solution viable dans cette période de crise sanitaire.»(Sujet 30). }\end{array}$} \\
\hline Décision inadéquate & 11 & $36,66 \%$ \\
\hline \multicolumn{3}{|c|}{$\begin{array}{l}\text { «Nous avons été surpris par la décision cruelle du ministère de l'enseignement supérieur, en effet en un temps } \\
\text { record presque deux semaines, nous sommes dans l'obligation de numériser nos supports du cours afin de migrer } \\
\text { d'un mode d'enseignement classique à un mode d'enseignement entièrement en ligne »(sujet 15). } \\
\text { «A mon avis c'est une décision hâtive... j'ai refusé de travailler en ligne car le module que j'enseigne nécessite } \\
\text { un contact direct avec les étudiants pour qu'ils puissent bien le saisir »(Sujet 9). }\end{array}$} \\
\hline Totale & 30 & $100 \%$ \\
\hline
\end{tabular}

Tableau 2. L'engagement des enseignants universitaires envers l'EAD

Concernant les questions «Avez-vous bénéficié d'une période de formation avant de pratiquer l'EAD ? Pensez-vous que les enseignants universitaires doivent bénéficier d'une période de formation avant de pratiquer l'EAD ?». Nous avons constaté que la plupart des universités ont fait des formations à distance à leurs enseignants mais le taux de participation des enseignants était très faible. En effet, $30 \%$ enseignants uniquement ont assisté à ces formations. Voici deux verbatim dans ce sens :

«...Puisqu'il s'agit d'une transition brutale, notre rectorat a organisé des formations à distance à travers des webinars pour la majorité des enseignants. De même l'UVT a crée des espaces du cours virtuels sur ses plateformes pour que les enseignants puissent partager leurs cours avec leurs étudiants et elle a créé aussi des adresses mails professionnels pour tous le staff éducatifs et les étudiants afin de profiter de la gratuité de services offerts par tous les opérateurs téléphoniques» (Sujet 8). 
«...Beaucoup de mes collègues n'ont pas assisté à ces formations puisqu'ils croient que cela les oblige à enseigner à distance et au début on était tous résistant au changement... Personnellement j'ai hésité» (Sujet 15).

Aux questions «Quels sont les types de supports de cours virtuels que vous utilisez lors de l'EAD ? Pourquoi ? », La plupart des enseignants tendent à employer systématiquement les documents papiers au profit des documents numériques. En effet, $70 \%$ des enseignants interviewés avancent que les supports fournis à leurs étudiants sont des supports statiques (PDF, WORD, des présentations PowerPoint..) alors que le reste utilise des cours multimédias (hypertexte, audio, vidéo...). Ce choix des supports statiques peut s'expliquer par la facilité de l'emploi de ce genre de support. Pour d'autres, la préparation des supports multimédias peut s'avérer pénible et nécessite une formation au préalable. Ceci met en évidence le manque des compétences numériques de certains enseignants.

Toutefois, 30\% enseignants interviewés maitrisent les technologies numériques et pensent que les supports multimédias des cours sont plus pratiques et plus efficaces dans le cadre de l'EAD.

Quels sont les types de supports de cours virtuels que vous utilisez lors de l'EAD ? Pourquoi ?

\begin{tabular}{|c|c|c|}
\hline \multicolumn{3}{|c|}{ Quels sont les types de supports de cours virtuels que vous utilisez lors de l'EAD ? Pourquoi? } \\
\hline & Nbre & $\%$ OBS \\
\hline La facilité de l'emploi des supports statiques & 14 & $46,66 \%$ \\
\hline \multicolumn{3}{|c|}{$\begin{array}{c}\text { «Nous sommes déjà habitués à utiliser les fichiers PDF et Power Point lors de l'enseignement en présentiel... } \\
\text { personnellement je n'ai jamais utilisé des cours multimédias auparavant »(Sujet 11). }\end{array}$} \\
\hline La difficulté de la préparation des supports multimédias & 7 & $23,33 \%$ \\
\hline \multicolumn{3}{|c|}{$\begin{array}{l}\text { «La réorganisation des contenus de mes papiers en cours multimédias n’est pas une tâche aisée pour moi, elle } \\
\text { demande beaucoup du temps et elle nécessite une préparation sérieuse » (Sujet 1). } \\
\text { «La préparation des cours spécifiques à l'enseignement virtuelle nécessite certainement une formation préalable... ca } \\
n e \text { se fait pas du jour au lendemain »(Sujet 9). }\end{array}$} \\
\hline L'efficacité et la praticité des supports multimédias & 9 & $30 \%$ \\
\hline \multicolumn{3}{|c|}{$\begin{array}{l}\text { "....j'aime bien donner mes cours sous formats vidéos ... généralement, ce format est plus pratique, il permet de } \\
\text { minimiser la quantité de travail envoyée aux étudiants...Je sais bien que la majorité de mes collègues veulent imiter ce } \\
\text { qu'ils font en classe mais il faut bien adapter leurs supports du cours au nouveau mode d'enseignement »(Sujet 21). } \\
\text { «J'aimerais être concis, je préfère donner à mes étudiants que les concepts clés et éviter la surcharge d'informations, } \\
\text { c'est pourquoi je vois que les cours multimédias sont les plus adéquats et les plus utiles» (Sujet 28). }\end{array}$} \\
\hline Totale & 30 & $100 \%$ \\
\hline
\end{tabular}

Tableau 3. Les types de supports de cours virtuels

Selon [LEW93], les plates-formes synchrones offrent la possibilité d'échanger des images de l'interlocuteur et de partager les tâches qu'il est en train de réaliser. Ce type de plate-forme contribue ainsi à enrichir la communication en réintroduisant les aspects non-verbaux dans la conversation. Mais malgré les multiples avantages des échanges synchrones, $60 \%$ des enseignants interrogés préfèrent le recours à la communication asynchrone. Pour eux, l'utilisation des plates-formes asynchrones comme l'envoi des courriels ainsi que le dépôt et le téléchargement de document sur Google Classroom, est simple à utiliser, laisse le temps de réflexion aux étudiants et favorise leur autonomie.

Par contre, 23,33\% des enseignants interrogés pensent que l'usage de la communication synchrone à l'égard de Teams, Zoom, Google Meet, est indispensable surtout pour le cas les cours de statistiques et de mathématiques. Le reste déclare que l'idéal est de combiner l'utilisation des plateformes synchrones et asynchrones à la fois. 
Quelles sont les plateformes que vous utilisez pour l'EAD ? Laquelle vous semble la mieux adaptée pour l'EAD ? Pourquoi ?

Nbre

$\%$ OBS

Les plates-formes asynchrones

18

$60 \%$

"Il est plus facile pour moi d'utiliser le Google Classroom par contre je ne maitrise pas bien l'application Zoom. Il convient donc de continuer à proposer un accompagnement et des formations aux enseignants pour les aider à se familiariser avec ces plates-formes synchrone»(Sujet 19).

«Je pense que les cours asynchrones (Google Classroom) donnent à mes étudiants plus de temps pour interagir et comprendre le contenu du cours en suivant leurs rythme d'apprentissage » (Sujet13)

\section{Les plates-formes synchrones} 7

$23,33 \%$

«Je suis un professeur d'économétrie, je ne peux pas me contenter par les plates formes asynchrones... j'utilise souvent les visioconférences sécurisés via le Google Meet»(Sujet1).

La combinaison des plates-formes synchrones et asynchrones 5

$16,66 \%$

«... pour qu'un cours soit intéressant, l'enseignant doit alterner les activités « synchrones » (en temps réel, à heure fixe) comme une séance sur Google Meet, et d'autres activités «asynchrones » (contenu préenregistré, exercices) comme des activités sur Google Classroom » (sujet 7).

\section{Totale}

Tableau 4. Les plateformes utilisées pour l'EAD

Concernant la question «Comment évaluez-vous votre expérience avec l'EAD ? », 63,33\% des répondants pensent que l'EAD est une expérience stressante, désagréable et pénible qui bouleverse les anciennes méthodes de travail. En effet, plusieurs enseignants se trouvent face à une situation pédagogique difficile, totalement nouvelle et différenciée. Ces enseignants ont ressenti le besoin d'apporter d'importantes adaptations pédagogiques à leurs cours et dans un temps record. Par contre, $36,66 \%$ des interviewés apprécient l'expérience de l'enseignement virtuel et croient qu'il s'agit bien d'une expérience avantageuse et enrichissante professionnellement. 
«La masse de travail semble plus élevée en passant d'un apprentissage en présentiel à un apprentissage à distance ...nous constatons que temps estimé pour préparer les cours en ligne est plus élevé que le temps consacré en classe, en effet mise à part le contenu du cours, l'enseignant doit accorder plus de temps pour animer les forums et répondre aux questions et aux commentaires des étudiants »(Sujet 27).

« Le charge de travail a doublé» (Sujet 1).

Expérience stressante 3

$10 \%$

"La transformation numérique qu'a connu le secteur de l'enseignement a bouleversé notre rythme de travail, une sorte de solitude vient de s'installer, un manque de contact physique et social est ressenti par les enseignants, ce qui nous stresse et démotive énormément» (Sujet 19)

\begin{tabular}{|c|c|c|}
\hline Expérience désagréable & 7 & $23 \%$ \\
\hline \multicolumn{3}{|c|}{ «Enseigner derrière un écran à partir de ma maison est vraiment désagréable» (Sujet 25). } \\
\hline Expérience enrichissante & 6 & $20 \%$ \\
\hline \multicolumn{3}{|c|}{$\begin{array}{c}\text { «Je suis plutôt satisfait de l'expérience d'enseignement virtuel, c'était vraiment une expérience enrichissante sur tous } \\
\text { les plans» (Sujet 18) } \\
\text { «J'apprécie bien mon expérience... Elle est très intéressante et enrichissante professionnellement» (Sujet 15). }\end{array}$} \\
\hline Expérience avantageuse & 5 & $16.66 \%$ \\
\hline \multicolumn{3}{|c|}{$\begin{array}{l}\text { «C'est une bonne expérience... Ce nouveau mode d'enseignement peut aider les étudiants introvertis à surmonter leur } \\
\text { timidité... l'EAD implique les étudiants dans l'animation du cours mieux que les séances en présentiel ... } \\
\text { «C'est une expérience positive ...les horaires sont plus flexibles et on peut dépasser les limitations de temps» (Sujet 5) }\end{array}$} \\
\hline Totale & 30 & $100 \%$ \\
\hline
\end{tabular}

Tableau 5. L'évaluation de l'expérience de l'EAD

\subsection{Difficultés liées à l'instauration de l'EAD durant le confinement}

Nos verbatim montrent que la résistance aux changements et la réticence que certains enseignants pourraient éprouver face à l'usage des nouveaux médias éducatifs $(26,66 \%)$ est le frein principal lié à l'instauration de l'EAD. Nous rejoignons ici les résultats de [CAN19] qui trouvent que les innovations technologiques peuvent provoquer une résistance au changement.

Ce frein est suivi par l'absence de lieu propice au travail à distance (20\%), la difficulté d'adaptation pédagogique lors de l'EAD (16,66\%) et la lenteur de la connexion de l'Internet en Tunisie $(13,33 \%)$. Le manque de motivation ainsi que le stress et l'anxiété pendant le confinement sont presque évoqués à parts égales (10\%). Le non maitrise de l'outil informatique $(6,66 \%)$ par certains enseignants et étudiants constitue également un frein non négligeable à l'instauration de l'EAD. En effet, la complexité perçue de certains instruments technologiques freine son adoption [CAN19].

Le dernier frein est le problème d'accès aux équipements de recherches en laboratoires, notamment pour les modules de sciences dures lors d'une séance des travaux pratiques $(3,33 \%)$. En effet, dans le domaine des sciences dures, les connaissances théoriques s'accompagnent souvent de connaissances pratiques et expérimentales dont la mise en accès global est difficile à opérer [PHI13].

De nos jours les systèmes électroniques donnent naissance à des technologies embarquées facilitant l'accès à des machines contrôlables, pilotables et manipulables à distance. Grand nombre de professeurs profite alors de ces avancées technologiques pour créer des laboratoires virtuels expérimentaux dans le but de réaliser des travaux pratiques à distance [CRA12, CLA 12].Cependant, 
dans un pays en voie de développement comme la Tunisie, il est complexe d'assurer des séances de TP à l'aide de ces systèmes électroniques.

Y a-t-il des obstacles qui vous freinent lors de l'EAD ? Lesquels ?

Résistance aux changements et la réticence

Nbre

$\%$ OBS

6

$20 \%$

«Pendant longtemps, j'agis dans le cercle fermé de mes étudiants. Aujourd'hui, on me demande brutalement d'ouvrir mes classes, de pratiquer de nouveaux modes d'enseignement, de montrer mes TD et mes cours. C'est dur psychologiquement de tout changer du jour au lendemain» (Sujet 6).

«Le saut est vraiment très grand surtout pour les enseignants qui délivraient le même cours depuis plus de dix ans» (Sujet 18).

\section{Problème de motivation}

3

$10 \%$

«Depuis la fermeture des universités, je me trouve désarmé pour faire travailler mes étudiants à distance. Susciter l'engagement et la motivation des étudiants dans une classe, est déjà une mission très difficile. A distance, c'est vraiment une gageure» (Sujet 30).

"Beaucoup des étudiants sont contre l'EAD, accroître la motivation des étudiants à l'égard de l'apprentissage en ligne est ainsi une mission très difficile voire même impossible pour certains» (Sujet 26).

\section{Stress et anxiété pendant le confinement}

3

$10 \%$

"Personnellement j'étais beaucoup stressée pendant la période de confinement j'avais une peur bleue de contracter le Coronavirus... je passais mon temps à faire le ménage et à tout désinfecter et je n'avais pas le goût ni le temps d'utiliser de nouveaux outils d'enseignement» (Sujet 3).

«La pandémie provoque sans doute beaucoup de stress et d'anxiété, durant le confinement je me souciais beaucoup de l'avenir incertain, je me déprimais carrément et je n'avais pas envie d'ouvrir mon PC pour pratiquer l'EAD »Sujet 5).

\section{Absence d'un espace propice à l'EAD}

$20 \%$

«C'est bien de garantir la continuité pédagogique durant le confinement mais personnellement mon appartement est petit et ma famille est assez nombreuse vraiment je n'ai pas un coin intime pour se concentrer et travailler à distance» (Sujet 15).

«Je n'arrive pas à faire des visioconférences en présence de mes enfants, ils font beaucoup de bruit et ils ne me laissent jamais tranquille pour faire un enseignement interactif de qualité ...Concilier confinement et enseignement à distance de chez soi au temps de Covid-19 avec les enfants à la maison est un vrai défi»( Sujet 27).

\section{Non maitrise de l'outil informatique} 2

"Certains outils comme les ressources audio, les visioconférences, le partage d'écran ou la classe virtuelle semblent très utiles aux enseignants mais souvent ils les maîtrisent mal ce qui les freinent dans leurs utilisations... même chose pour certains étudiants qui ne sont pas automnes dans leur utilisation des technologies»(Sujet 3).

\section{Difficulté pédagogique rencontré lors de l'EAD}

5

$16,66 \%$

«D'un point de vue pédagogique, L'EAD est très différent de l'enseignement en présentiel ... c'est très difficile de gérer une classe en ligne...Je dois changer totalement la pédagogie dont j'ai pratiquée pendant des années dans les classes fermées» (Sujet 17).

\section{Lenteur de la connexion de l'Internet}

"En Tunisie, l'EAD est extrêmement contraignant surtout avec la lenteur de connexion et les pannes répétés d'Internet, .... je n'arrive pas à faire mes vidéoconférences convenablement, si je possède une connexion surement mes étudiants ne le possèdent pas » (Sujet 9).

Problème d'accès aux équipements de recherche en laboratoire 1 $3,33 \%$

«L'EAD ne peut pas être appliqué dans tous les sciences ...pour réaliser des expériences pendant les séances de TP en chimie ou en biologie j'ai besoin certainement d'un laboratoire équipé de divers matériels, solutions chimiques et instruments de mesures » (Sujet 22)

\section{Totale}




\subsection{L'effet de l'EAD sur les inégalités sociales}

En ce temps de pandémie de Covid-19, les mesures d'EAD risquent de compromettre le droit d'éducation des étudiants qui font partie de familles modestes. En effet, notre étude montre que plus de la moitié des enseignants interviewés affirment que le nouveau mode d'apprentissage aggrave les inégalités sociales à la fois du côté des enseignants (vacataires ou contractuels) et des étudiants surtout ceux qui sont défavorisés et qui habitent dans des zones rurales.

Ces inégalités sont non seulement injustes et inacceptables par la majorité des enseignants, mais elles représentent indéniablement un obstacle à la migration vers un nouveau mode d'enseignement et à la démocratisation de l'enseignement supérieur.

\begin{tabular}{|c|c|c|}
\hline \multicolumn{3}{|c|}{$\begin{array}{l}\text { Pensez-vous que la décision de recourir à l'EAD en période de Covid-19 n'a pas pris en compte les } \\
\text { conditions sociales de certains étudiants et /ou enseignants? Comment? }\end{array}$} \\
\hline & Nbre & $\% \mathrm{OBS}$ \\
\hline L'EAD : cruel accélérateur des inégalités sociales & 18 & $60 \%$ \\
\hline \multicolumn{3}{|c|}{$\begin{array}{l}\text { "lors d'une séance de cours virtuelle, le taux de participation des étudiants est trop faible, puisque la majorité de } \\
\text { mes étudiants et surtout les brillants font parties des familles modestes. En effet, il est évident qu'entre un } \\
\text { étudiant qui possède un ordinateur portable personnel et une imprimante et celui qui ne possède qu'un vieil } \\
\text { ordinateur qu'il partage avec ses frères et soeurs, l'inégalité d'accès aux ressources en ligne semble très forte... } \\
\text { je vois que l'EAD approfondit l'inégalité sociale et l'équité de chance entre les étudiants»(Sujet 25) }\end{array}$} \\
\hline \multicolumn{3}{|c|}{$\begin{array}{c}\text { "En tant qu'enseignant vacataire, j'ai des difficultés financières énormes pour poursuivre mes séances de TD de } \\
\text { deuxième semestre puisque je ne possède pas à mon lieu de résidence une connexion Internet, je n'ai pas les } \\
\text { équipements informatiques nécessaires et ni un forfait téléphonique illimité pour maintenir le lien avec mes } \\
\text { étudiants »(Sujet 7) }\end{array}$} \\
\hline \multicolumn{3}{|c|}{ «Je pense que la crise sanitaire a révélé et accentué les inégalités et la fragilité de nos sociétés» (Sujet 4) } \\
\hline \multicolumn{3}{|c|}{$\begin{array}{c}\text { Afin de faciliter l'apprentissage des étudiants les plus défavorisés, il est préférable de combiner le présentiel et } \\
\text { l'EAD, car le passage à l'EAD est pour les étudiants les plus défavorisés injuste et même criminel, il ne fait } \\
\text { qu'aggraver les inégalités sociales»(Sujet 2) }\end{array}$} \\
\hline L'EAD n'approfondit pas les inégalités sociales & 12 & $40 \%$ \\
\hline \multicolumn{3}{|c|}{$\begin{array}{l}\text { «Nos étudiants sont tous des digitaux natives, et ils possèdent tous des comptes dans presque tous les réseaux } \\
\text { sociaux, sauf que lorsqu'il s'agit d'apprentissage et des devoirs à faire, ils invoquent de faux prétextes pour ne } \\
\text { pas assister aux cours virtuels »(Sujet 11). }\end{array}$} \\
\hline Totale & 30 & $100 \%$ \\
\hline
\end{tabular}

Tableau 7. L'effet de l'EAD sur les inégalités sociales

\subsection{Les perspectives futures de l'EAD}

En ce qui concerne les perspectives futures de l'EAD, 73,33\% des répondants considèrent que l'enseignement traditionnel en classe est irremplaçable, tandis qu'une minorité des enseignants interviewés considèrent que l'enseignement en ligne est un nouveau paradigme pédagogique qui devrait être généralisé dans l'avenir.

Nos résultats rejoignent alors ceux de [RES91] et [PER91]qui considèrent l'apprentissage comme un processus social dans lequel les interactions avec l'autre assument un rôle crucial. Dans ce sens, un cadre qui contraindrait les interactions avec l'autre et qui priverait des aspects non verbaux (regards, gestes...), comme c'est le cas de certaines formes de l'EAD, aurait peu de chance de conduire à un apprentissage efficace. En effet, l'apprentissage à distance, même quand elle véhicule l'image, conduit à des modalités d'échanges appauvries par rapport à l'enseignement présentiel. 
Pensez-vous que l'EAD est un nouveau paradigme pédagogique qui doit être généralisé dans les années futures?

\section{Pourquoi ?}

Nbre

$\%$ OBS

L'enseignement en présentiel est irremplaçable

22

$73,33 \%$

«A mon avis, l'enseignement en ligne ne peut en aucun cas remplacer l'enseignement en classe, le contact direct avec l'étudiant, les mimiques, le regarde dans les yeux et les gestes de l'enseignant sont très importants dans le passage optimale de l'information et dans la fluidité de la communication... L'expérience a révélé que l'EAD est inefficace» (Sujet 10).

«Pour obtenir de bons résultats, il faut combiner la communication verbale et non verbale. Toutefois, l'EAD tue la communication non verbale or cette dernière est un outil essentiel dans l'enseignement car elle affecte le sens et la qualité de discours transmis... Je vois que l'EAD dégrade la qualité de l'enseignement» (Sujet 22).

L'EAD : un nouveau paradigme à généraliser dans l'avenir 8 $26,66 \%$

«La situation ne va changer du jour au lendemain, l'EAD devra certainement être généralisé dans l'avenir. D'ailleurs c'est la meilleure solution en cas de crise pour assurer la continuité pédagogique» (Sujet 18).

« La grande révolution pédagogique dont rêvaient depuis des années certains enseignants doit avoir lieu, nous devons abandonner les méthodes classiques d'enseignement et instaurer le digital dans notre système éducatif» (Sujet 11).

\section{Totale}

Tableau 8. L'EAD : un nouveau paradigme d'apprentissage

\section{6. conclusion : Apports et limites de la recherche}

La crise sanitaire du coronavirus a fait subir aux systèmes éducatifs un choc sans précédent dans l'histoire de l'enseignement, en bouleversant la vie éducative sur tous les continents du monde.

La fermeture des lieux d'apprentissage présentiel et le passage rapide sans préparation à l'apprentissage virtuel a largement perturbé les étudiants et les enseignants. De nombreux étudiants et enseignants font face à des contraintes qu'ils n'avaient jamais connues auparavant. Même si les étudiants sont nés avec les outils numériques entre les mains, et que certains enseignants sont formés pour utiliser les plateformes en ligne, l'expérience de l'EAD pose certainement plusieurs défis tant pour les étudiants que pour les enseignants.

Dans cet article, nous avons essayé d'explorer l'expérience de l'enseignement en ligne dans les universités tunisiennes pendant la pandémie de Covid-19, de comprendre les freins qui entravent les enseignants lors de l'EAD et de mettre la lumière sur l'effet de ce dernier sur le processus d'apprentissage et sur l'inégalité sociale. Pour ce faire, nous avons réalisé une étude qualitative exploratoire via des entretiens individuels semi-directifs menés à l'aide d'un guide d'entretien auprès de trente enseignants opérant dans des différentes universités tunisiennes.

Nos résultats montrent que l'apprentissage en présentiel offre des possibilités d'interaction entre l'enseignant et les étudiants qu'il est difficile de reproduire à distance, notamment avec une formation déficiente. En effet, plusieurs enseignants universitaires ont rencontré maintes difficultés lors de l'EAD, à savoir : des difficultés d'ordre psychologique (la résistance aux changements et la réticence que certains enseignants éprouvent à l'égard de l'usage des nouveaux outils éducatifs, le manque de motivation ainsi que le stress et l'anxiété pendant le confinement), pédagogique (la difficulté d'adaptation pédagogique lors de l'EAD), technique (la non maitrise de l'outil informatique ainsi que le problème d'accès aux équipements de recherche en laboratoire pour certains matières en sciences « dures » lors de déroulement d'une séance des travaux pratiques) et matériel (l'absence de lieu propice au travail à distance et la lenteur de la connexion de l'Internet). 
Alors que les universités sont contraintes plus que jamais, au temps de la Covid-19, d'adapter très rapidement leurs cours pour les maintenir à distance, il semble que l'égalité sociale et l'équité de chance d'apprentissage sont sérieusement compromises. En effet, notre étude qualitative montre que le recours à l'EAD risque d'amplifier les inégalités sociales et l'équité des chances à la fois du côté des enseignants et des étudiants, des inégalités territoriales dans l'accès aux technologies dans les familles modestes. Ces inégalités sont inacceptables par la plupart des enseignants et elles sont indéniablement un obstacle à la démocratisation de l'enseignement supérieur.

Ces résultats peuvent certainement être améliorés si nous avions impliqué les étudiants dans notre étude exploratoire. En plus, les résultats dégagés de la présente recherche ne peuvent faire l'objet d'une généralisation absolue. Pour pallier cette limite, une recherche quantitative devrait envisager, dans des voies futures de recherche, à une enquête nationale portant sur l'implication à la fois des enseignants et des étudiants dans l'EAD.

Les résultats de cette étude comportent aussi des implications pour les décideurs politiques en rapport avec l'enseignement supérieur et la formation continue. En effet, il est recommandé aux ministères de l'éducation, de l'enseignement supérieur et de la recherche scientifique ainsi que le ministère de la télécommunication d'investir dans ce nouveau paradigme d'apprentissage en améliorant les infrastructures via l'installation des fibres optiques, la mise en place de 5G, l'intensification et la généralisation des équipements digitaux dans les établissements scolaires et universitaires.

Toutefois, nous ne pouvons pas nier l'agilité et la réactivité des enseignants universitaires tunisiens face à ces conditions inédites. Cette nouvelle expérience aura sans aucun doute un impact positif sur l'amélioration du secteur d'enseignement universitaire tunisien dans les plus prochaines années.

\section{Bibliographie}

[ABI 13]ABID-ZARROUK, S. B., Efficacité de l'enseignement à distance : mythe ou réalité? paris : l'harmattan. 2013.

[ABI 15]ABID-ZARROUK, S. B. „Les efficacités technologiques éducatives dans S. B. AbidZarrouk (dir.), Estimer l'efficacité en éducation (p. 215-228). Paris : L'Harmattan, 2015.

[ADE 11]ADEMILDE SILVEIRA,S, « Le design pédagogique et les modes d'interaction dans l'éducation à distance » ,distances et savoirs, Vol. 9, n², pp. 219-233, 2011.

[AND 05]ANDREANI J.C. et CONCHON F. «Fiabilité et validité des enquêtes qualitatives. Un état de l'art en marketing », Revue Française du Marketing, Mars, 201, pp 5-22, 2005.

[AND 13] ANDERSON, W, Independent learning. Dans M. G. Moore (dir.), Handbook of distance education (p. 86103). New York, NY : Routledge, 2013.

[ATT 20] ATTEBERRY, A. \& MCEACHIN, A. «School's out: the role of summers in understanding achievement disparities». American educational research journal, 2020.

[BAT 18] BATES, T.« The 2017 national survey of online learning in Canadian post secondary education: Methodology and results ».International Journal of Educational Technology in Higher Education, 15(29), pp 1-17,2018.

[BEA 16] BEAUCHESNE A. ET DEAUDELIN C., « Réussir l'implantation d'une innovation en formation à distance : à la recherche des conditions propices », Open Editions Journals Tréma, n44, pp.7-24, 2016.

[BLA 04]BLANDIN, B. „«Historique de la formation ouverte et à distance », in actualité de la formation permanente $\mathrm{n}^{\circ} 189$, Mars-Avril 2004, pp.69-71, 2004.

[BON 10]BON, A., «Information and communication technologies in tertiary education in subSaharan Africa » .dans D. Teferra , \& H. Greijn, «Enseignement supérieur et mondialisation Défis, menaces et opportunités pour 1'Afrique » (p. 135). Centre de coopération internationale pour le développement universitaire de lee Université de Maastricht (MUNDO),2010.

[BUR 20] BURGESS, S. \& SIEVERTSEN, H. H., «Schools, skills, and learning: The impact of COVID-19 on education. »,CEPR Policy Portal. Retrievedfromhttps://voxeu.org/article/impact-covid-19-education, 2020.

[CAC 06] CACHALDORA, C. «Les TIC à l'aide des scientifiques du Sud ». Revue électronique internationale , ${ }^{\circ} 2$, pp.31-44, 2006.

(C) 2021 ISTE OpenScience - Published by ISTE Ltd. London, UK - openscience.frPage | 16 
[CAN 19]CANEVA, C. « Facteurs d'adoption ou de rejet des technologies chez les professeurs-formateurs d'enseignants : le cas de l'université costaricienne. »International Journal of Technologies in Higher Education. Vol.16, n ${ }^{\circ}$, pp.15$35,2019$.

[CAR 20] CARVALHO, S., ROSSITER, J., ANGRIST, N., HARES, S. \& SILVERMAN, R. «Planning for School Reopening and Recovery After COVID-19.»Washington. Center for Global Development. https://www.cgdev.org/publication/planning-school-reopening-and-recovery-after-covid-19, 2020.

[CHR 12]CHRISTIAN, D. (2012). « Les pays en développement à l'ère de l'e-Learning », Unesco, Institut international de planification de l'éducation.

[CLA 12] CLAESSON L, HAKANSSON L «Using an online remote laboratory for electrical experiments in upper secondary education ». International Journal of Online Engineering, vol. 8, Special Issue 2, p. 24-30, 2012.

[CRA 12] CRABEEL N, CAMPOS NEVES B, MALHEIRO B, «Remote Labs Platform ». International Journal of Online Engineering, vol. 8, Special Issue 2, p. 13-18, 2012.

[DAN 96] DANIEL, J. Mega-universities and knowledge media: Technology strategies for higher education, London: Kogan, 1996.

[FEN 18] FENDLER, R. J., RUFF, C. ET SHRIKHANDE, M. M. «No significant difference ».Online Learning, 22(1), 39-60, 2018.

[GAR 13] GARRISON, D. R. ET AKYOL, Z. (2013). The community of inquiry theoretical framework. Dans M. G. Moore (dir.), Handbook of Distance Education (p. 104-119). New York, NY : Routledge.

[GAV 08]GAVARD-PERRET M.L., GOTTELAND D., HAON C. et JOLIBERT A., Méthodologie de la recherche: Réussir son mémoire ou sa thèse en sciences de gestion. Pearson education france, paris, 2008.

[HAE 20] HAECK, C. \& LEFEBVRE, P. «Pandemic School Closures May Increase Inequality in Test Scores » .Canadian Public Policy, ${ }^{\circ} 46$ vol .1,pp82-87,2020.

[HAM 11] HAMMES-ADELÉ SONIA. Traduction temporelle de la relation humain-technologie-organisation : Validation et perspectives autour de la symbiose, Thèse de Doctorat en Psychologie, Université Paul Verlaine Metz,2011.

[IFE 10] IFERGAN, P. \& BESCOS, P. L., «Les facteurs objectifs de la complexité de la tâche en audit légal objective ». Actes du 3lème Congrès de l'AFC, Nice,2010.

[JAO 04]JAOUEN, A. \&NAKARA, W. «Les systèmes d'information en microfirme : une approche par le bricolage organisationnel», Revue Internationale P.M.E. vol. 27, n 3-4, pp. 225-260, 2014.

[JOH 14] JOHNSON, S. « Applying the seven principles of good practice: Technology as a lever in an online research course. »Journal of Interactive Online Learning, 13(2), pp.41-50,2014.

[KEE 96] KEEGAN, D.Foundations of distance education . London and New york: Routledge (3rd. ed.), 1996.

[KAR 12] KARSENTI T, GARRY RAYMOND-P, BENZIANE A, NGOY-F, BALTHAZAR B et BAUDOT F .La formation de formateurs et d'enseignants à l'ère du numérique : stratégies politiques et accompagnement pédagogique, du présentiel à l'enseignement à distance. Montréal : Réseau international francophone des établissements de formation de formateurs (RIFEFF) / Agence universitaire de la Francophonie (AUF),2012.

[KUH 20] KUHFELD, M., JOHNSON, A., SOLAND, J., RUZEK E., TARASAWA, B. et LIU, J. Projecting the potential impacts of COVID-19 school closures on academic achievement. (Ed Working Paper: 20-226). Retrieved from Annenberg Institute at Brown University,2020.

[LEE 17] LEE, K. « Rethinking the accessibility of online higher education: A historical review». Internet and Higher Education, 33, pp15-23,2017.

[LEW 04] LEWIS C. et HEDEGAARD T. «Online education : issue and some answers ». T.H.E. Journal, pp. 68$71,1993$.

[LYY 01] LYYTINEN, K., \& DAMSGAARD, J. « What's wrong with the Diffusion of Innovation Theory ». IFIP Conference Proceedings, 187, pp173-190,2001.

[MAR 18] MARZOUK , H , «Fuite des cerveaux : quand les compétences quittent le pays », l'économiste magrébin,2018 .

[MER 14] MERRILL, M. D. ET ELEN, J. « A look forward«. dans J. M. Spector et al.. (dir.), Handbook of research on educational communications and technology (pp. 873-874).New York, NY : Springer Science\&Business,2014. 
[MOO 13] MOORE, M. G. «The theory of transactional distance». dans M. G. Moore (dir.), Handbook of distance education (pp. 66-85). New York, NY : Routledge,2013.

[MOO 91] MOORE, G.C. ET BENBASAT, I. « Development of an Instrument to Measure the Perceptions of Adopting an Information Technology Innovation ». Information Systems Research, vol`2, n³, pp. 192-222, 1991.

[PER 91] PERRET-CLERMONT A.N., J.F. et BELL N.. The social construction of meaning and cognitive activity in elementary school children. In : L.B. Resnick, J.M. Levine et S.D. Teasley (Eds) Perspectives on socially shared cognition. Washington D.C. : American Psychological Association, 1991.

[PHI 13]PHILIPPE L , DENIS B , SERGE B \& RAPHAËL N , «LABENVI, un dispositif pour les travaux pratiques à distance . Présentation et analyse des nouveaux usages ». Interfaces numériques. Vol 2 - n 3,pp. 453-467,2013.

[POU 10]POURTIER R., «L'éducation, enjeu majeur de l'Afrique post- indépendances : Cinquante ans d'enseignement en Afrique : un bilan en demi-teinte », Revue Afrique contemporaine, Vol.3 n²35, pp. 101-114, 2010.

[POU 15]POURCELOT, C. ET ABID-ZARROUK, S. B.«Construire des outils d'évaluation de l'efficacité d'un dispositif pédagogique à distance : le cas de l'efficacité interne d'IFADEM RDC/Katanga ». Distances et médiations des savoirs, 3(11), pp 1-23,2015.

[POW 02]POWER, M..«Générations d'enseignement à distance, technologies éducatives et médiatisation de l'enseignement supérieur ». Revue de l'éducation à distance. Vol. 17, nº 2, pp.57-69,2002.

[PRE 95] PRESCOTT, M. B.,\& CONGER, S. A. «Information Technology Innovations: A Classification by IT Locus of Impact and Research Approach». Data Base Advances, 26 (2-3), pp20-41,1995.

[PSA 20] PSACHAROPOULOS, G., PATRINOS, H., COLLIS, V.\& VEGAS, E. The COVID-19 cost of school closures. https://www.worldbank.org/education/covid-19-cost-school-closures, 2020.

[RES 04]RESNICK L.B. "Shared cognition : thinking as a social practice ». cite dans : Resnick L.B., Levine J.M. \&Teasley S.D. (Eds) Perspectives on socially shared cognition. Washington, D.C. :American Psychological Association, 1991.

[ROG 03]ROGERS, E. M. Diffusion of innovations (5e éd.). New York, NY : The Free Press,2003.

[ROG 95] ROGERS, E..Diffusion of innovations. New York: Free Press (4th edition),1995.

[RUM 97] RUMBLE, G. Costs and economics of open and distance learning. London and stirling: uk: kogan,1997.

[TED 07] TEDDLIE, C., \& YU, F. «Mixed methods sampling: A typology with examples ». Journal of Mixed Methods Research, 1(1), pp. 77-100,2007.

[VAN 20] VAN LANCKER, W. \& PAROLIN, Z. « COVID-19, school closures, and child poverty: a social crisis in the making. », The Lancet Public Health, 5(5), pp.243-244,2020.

[VEN 02]VENDRAMIN, P. ET VALENDUC, G. La diffusion des innovations : le cas Internet, Centre de recherche Travail \& Technologies, Lettre EMERIT, $\mathrm{N}^{\circ} 32$, pp.1-8,2002.

[ZAN 17] ZANCARO, A., NUNES, C. S. ET DOMINGUES, M. J. C. « Evaluation of free platforms for delivery of massive open online courses (MOOCs) ». Turkish Online Journal of Distance Education, 18(1), pp166-181,2017. 


\section{Entretien à l'intention des enseignants}

Comment jugez-vous la décision du ministère de l'enseignement supérieur de recourir à l'EAD pendant la pandémie de Covid-19 ? Est-ce que vous avez pratiqué l'EAD pendant cette période ?

\section{Expérience avec l'EAD}

Avez-vous bénéficié d'une période de formation avant de pratiquer l'EAD ?

Pensez-vous que les enseignants universitaires doivent bénéficier d'une période de formation avant de pratiquer l'EAD?

Quels sont les types de supports de cours virtuels que vous utilisez lors de l'EAD ? Pourquoi ?

Quelles sont les plateformes que vous utilisez pour l'EAD ? Laquelle vous semble la mieux adaptée pour l'EAD ? Pourquoi ?

Comment évaluez-vous votre expérience avec l'EAD ?

Y a-t-il des obstacles qui vous freinent lors de l'EAD ? Lesquels ?

Les freins de l'EAD

L'effet de l'EAD sur les inégalités sociales

Pensez-vous que la décision de recourir à l'EAD en période de Covid-19 n'a pas pris en compte les conditions sociales de certains étudiants et /ou enseignants? Comment ?

Pensez-vous que l'EAD est un nouveau paradigme pédagogique qui doit être généralisé dans les années futures? Pourquoi ?

\section{Fiche signalétique :}

Genre : $\square$ Féminin $\square$ Masculin

Age : $\square$ Moins de 27 ans

Statut : $\square$ Professeur

Maître Assistant

$\square$ Enseignant contractuel
De 27 ans à 35ans

$\square .36$ ans à 45 ans

Maître de conférences

Assistant

Enseignant Vacataire

Catégorie discipinaire :

Droit, Economie, Gestion, Sciences politiques

Arts, Langues, Lettres, Sciences humaines

Sciences de l'ingénierie et technologies

Santé et biologie 